\title{
PLASMA-ETCHED NEURAL PROBES
}

\author{
David T. Kewley', Matthew D. Hills ${ }^{2}$, David A. Borkholder ${ }^{2}$, Ion E. Opris ${ }^{2}$, Nadim I. Maluf ${ }^{2}$, \\ Christopher W. Storment ${ }^{2}$, James M. Bower ${ }^{1}$, and Gregory T. A. Kovacs ${ }^{2}$ \\ ${ }^{1}$ Computation and Neural Systems, MC 216-76 \\ California Institute of Technology \\ Pasadena, CA 91125 \\ ${ }^{2}$ Center for Integrated Systems, CISX 202 \\ Stanford University \\ Stanford, CA 94305-4075
}

\begin{abstract}
This paper presents a new method for microfabricating silicon-based neural probes that are designed for neurobiology research. Such probes provide unique capabilities to record high-resolution signals simultaneously from multiple, precisely defined locations within neural tissue. The fabrication process utilizes a plasma etch to define the probe outline, resulting in sharp tips and compatibility with standard CMOS processes. A low-noise amplifier array has been fabricated through the MOSIS service to complete a system that has been used in multiple successful physiological experiments.
\end{abstract}

\section{INTRODUCTION}

It is necessary to study the simultaneous functioning of many neurons in order to understand how the nervous system works [1]. Until now, most investigations into the functioning of groups of neurons have been conducted by studying one neuron at a time, then pooling the results into a summary that has been assumed to represent the activity of an entire population of cells. By using multiunit recording techniques, however, it has become clear that correlations among the activities of neurons often carry information that cannot be found by studying neurons separately. For instance, a recent study in the olfactory bulb focused on the considerable variability seen in the responses of individual mitral cells to odor stimulation [2]. With a single-neuron recording, this variability would have appeared random, but simultaneous recordings revealed it to be coordinated among several cells. In many other applications, it has been found to be crucial to study multiple cells simultaneously (e.g., $[3,4]$ ).

Of all the available techniques for studying neural function, it appears that only arrays of extracellular microelectrodes have the ability to record the activities of many individual neurons simultaneously, on a fast time scale, and in intact, behaving animals. Extracellular arrays are most commonly made from fine wires [2-4]. Wire arrays are attractive to the neurophysiologist because they are dependable. They can be built in the laboratory using readily available materials, and they have been demonstrated by many researchers to be capable of acquiring high-quality data.

Micromachined neural probes readily offer several advantages over wire arrays, including: (1) individual shafts easily support multiple electrodes, which facilitates the study of local networks; (2) relative electrode locations are precise and repeatable; and (3) probe shafts displace less volume than wire bundles, causing less neural damage.

A longer-term advantage offered by silicon neural probes is the possibility of integrating circuits directly on the probes. Neurophysiologists are pushing the technology toward a greater number of electrodes per array [2-4], and are hoping to eventually use systems with hundreds of channels. As the number of electrodes increases, it becomes nontrivial to make connections to the electrodes. Active probes with integrated amplifiers and multiplexers will be necessary to keep the packaging problem tractable, particularly in mobile, chronically implanted animals.

Microfabricated neural probes were first developed in the late 1960s [5]. Since then, several groups have developed neural probes [6-13], including two that have integrated circuitry $[6,13,14]$. Almost all of these previous efforts have produced probes with cross-sectional dimensions near the electrodes greater than $100 \mu \mathrm{m}$, which is likely to result in significant damage to the nearby neurons. Only two $[6,7]$ have produced probes with properties that appear to be suitable for use in basic neuroscience.

The defining step in any neural probe fabrication process is the probe shaping technique. Of the two previous highquality probe fabrication efforts, one uses a diffused $\mathrm{p}++$ etch stop together with an anisotropic wet etch to define the probe shape [6]. The other uses (110) oriented wafers and an anisotropic wet etch, stopping on the $\{111\}$ planes, to define the shaft sidewalls [7]. Both processes require extra steps specifically to produce sharp tips, and both require significant CMOS process modifications to incorporate circuits.

Neurons communicate principally via transient $(\approx 1 \mathrm{~ms})$ electrical signals called action potentials. When recorded extracellularly, these events occur as potentials with typical amplitudes up to $500 \mu \mathrm{V}$, and have significant power in a frequency band from tens or hundreds of $\mathrm{Hz}$ up to several $\mathrm{kHz}$. A field potential is another, lower-frequency, signal that is also often of interest to neurobiologists. These signals typically have significant power from below one $\mathrm{Hz}$ up to hundreds of $\mathrm{Hz}$, and can range in amplitude up to a few $\mathrm{mV}$.

In order to record from a relatively intact neural network, an electrode array must be designed to minimize the damage it inflicts when it is inserted into the brain. The electrodes should be constructed of biocompatible materials, particularly if they will be implanted chronically. The electrode shafts should be straight, smooth, and stiff, and have sharp tips and small crosssections. The shafts should be aligned with the insertion device so that they are inserted straight into the tissue [15]. These geometrical and mechanical constraints are particularly important when recording from the vertebrate brain, which is wrapped in a membrane called the pia mater. If the electrode dimples (locally depresses) the brain during penetration of the pia, the superficial neural tissue will probably be damaged. 


\section{SYSTEM DESIGN}

The goal of this work has been to develop a complete neural probe system and to make it available to the research community. To facilitate this development, the probe and circuit fabrication have been pursued independently, with care taken to preserve the ability to integrate the two in the future. This has meant keeping all processing temperatures at or below $300^{\circ} \mathrm{C}$.

Probe Design. The current probes are fork shaped, having one or more parallel shafts projecting from a base (Fig. 1). Current designs support 16 iridium electrodes placed near the tips of the shafts, with a single reference electrode near the midpoint of the middle shaft.

The electrical properties are primarily determined by the electrode characteristics [16]. The electrode design is based upon previous research [18].

Plasma etching provides the flexibility of defining arbitrary probe outlines. A second important feature is the low curvature in the probe shafts. Najafi, et al., accomplished this by depositing alternating layers of insulators with opposite stresses [6]. In the present design, a low-stress nitride has been used, eliminating the need for stress cancellation. Other advantages of the nitride are that it is deposited at low temperatures $\left(300^{\circ} \mathrm{C}\right)$, it is very conformal, and it has an extremely low pinhole density.

The use of two metal layers-one for traces, the other for the electrodesminimizes the shaft cross-section. Another benefit of this approach is the flexibility to place the electrodes at arbitrary positions on

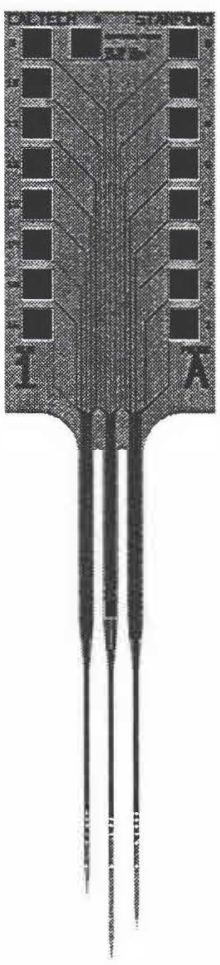

Figure 1. Plan view of a probe. allowing the cutoff to vary between $1 \mathrm{~Hz}$ and $10 \mathrm{kHz}$.

A prototype board was made to mount the preamplifierarray chip directly to the probe carrier printed circuit board. A separate signal-conditioning amplifier was built using commercial components. It provides a gain of 2.8 to match signal levels to the input range on the data acquisition system. This amplifier also provides a two-pole rolloff at $3 \mathrm{kHz}$ for anti-aliasing.

\section{PROBE FABRICATION}

Starting with standard 4-inch, (100)-oriented, p-type wafers, the probe fabrication process proceeds in three phases (Fig. 3):

1. Thin-film deposition (electrodes, bond pads, and insulated interconnect traces)

2. Probe-shaping etches

3. Finishing steps

Thin Film Deposition. Initially, a $1 \mu \mathrm{m}$ insulation layer of silicon nitride was deposited in a PECVD system (STSElectrotech) using dual-frequency RF excitation of the plasma. Process parameters were adjusted to yield low stress (typically $<100 \mathrm{MPa}$ tensile).

The first metal layer was then deposited and patterned using a liftoff process. After defining the liftoff structure $(1 \mu \mathrm{m}$ of photoresist over $500 \mathrm{~nm}$ of polysilicon), $25 \mathrm{~nm}$ of titanium (to improve adhesion) and $500 \mathrm{~nm}$ of gold were sequentially deposited by electron-beam evaporation. The liftoff was accomplished by dissolving the photoresist in acetone, and the polysilicon was plasma etched with $50 \mathrm{sccm} \mathrm{SF}_{6}$ and $50 \mathrm{sccm}$ $\mathrm{C}_{2} \mathrm{ClF}_{5}$ at $260 \mathrm{~mW} / \mathrm{cm}^{2}$ and $150 \mathrm{mTorr}$.

The second layer of silicon nitride, $0.7 \mu \mathrm{m}$ thick, was deposited using the PECVD process. Contact holes to the bond pads and for gold-iridium contacts were etched in the nitride using a plasma with $50 \mathrm{sccm} \mathrm{SF}_{6}$ and $50 \mathrm{sccm} \mathrm{C}_{2} \mathrm{ClF}_{5}$ at $270 \mathrm{~mW} / \mathrm{cm}^{2}$ and $150 \mathrm{mT}$ Torr.

Another liftoff process was used to pattern the iridium as it was deposited, using a bilayer of photoresist over aluminum. A $75 \mathrm{~nm}$ layer of silicon nitride was deposited to avoid the formation of unwanted intermetallics between the aluminum and the gold exposed in the contact cuts. A $500 \mathrm{~nm}$ thick layer of aluminum was sputter deposited, followed by $1 \mu \mathrm{m}$ layer of spun-on photoresist that was then patterned. The aluminum was etched using a standard wet etch until the undercut was 1.5$2.0 \mu \mathrm{m}$. Finally, the thin nitride layer was removed from the contact holes with the nitride plasma etch.

The wafers were placed in a custom magnetron sputtering system to deposit $300 \mathrm{~nm}$ of iridium after a $30 \mathrm{~nm}$ tacking layer of chromium. To lift off the unwanted $\mathrm{Cr} / \mathrm{Ir}$, the wafers were reference electrode input. The input range is sufficient accommodate the range of signals expected $(10 \mu \mathrm{V}-5 \mathrm{mV})$. The passband gain of 150 is provided in three stages. The output buffer, B, was designed to drive a $10 \mathrm{nF}$ load. The output is fed back into A3 to set the DC output level 10 an on-chip reference value. The low pass cutoff is fixed at $35 \mathrm{kHz}$ by the shunt capacitor between A3 and B. The coupling capacitors in combination with M1-M4, which are biased in the sub-threshold regime, create the 2 pole high pass filter. The hpf signal is derived from a chip input,

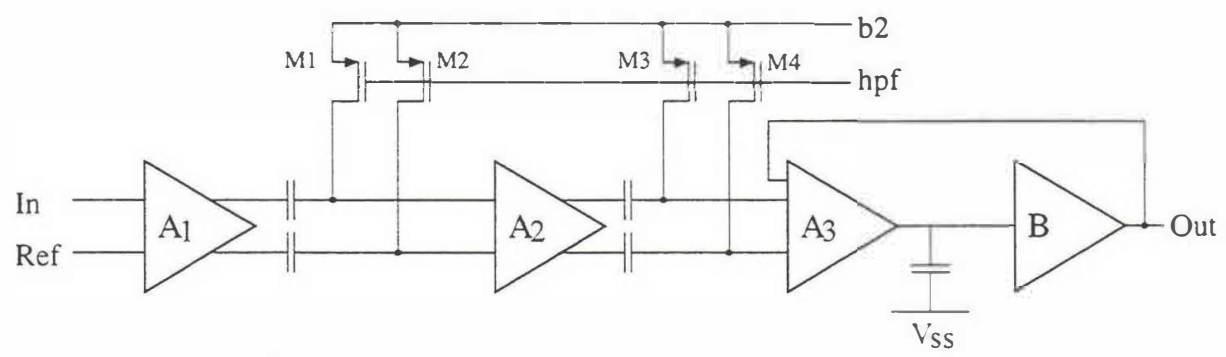

Figure 2. Schematic of one channel of the preamplifier circuit showing the filtering elements. 


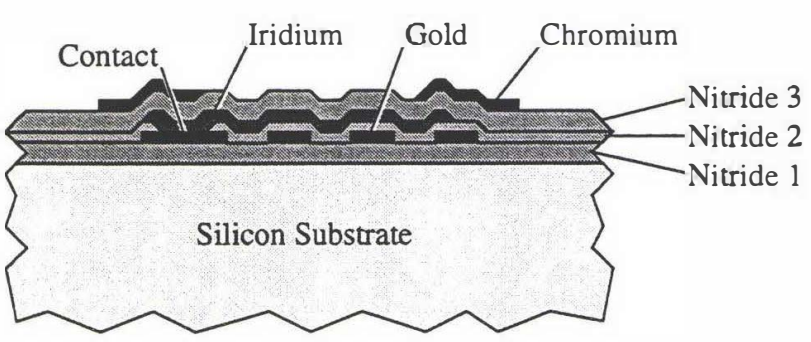

(a)

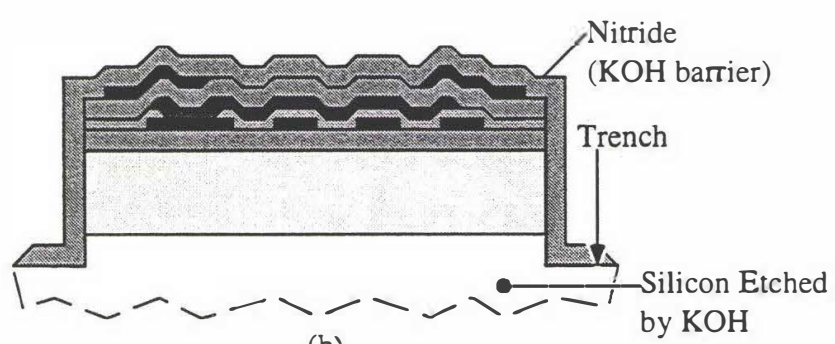

(b)

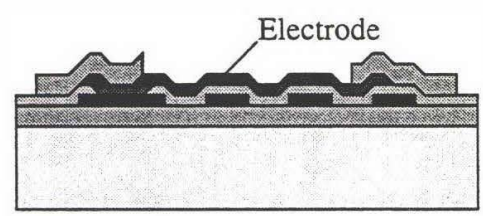

(c)

Figure 3. Schematic representation of the probe fabrication process flow, seen in a cross-section through an electrode site (not to scale). Parts (a), (b), and (c) show the end of the three major processing phases discussed in the text.

placed in a fresh solution of sulfuric acid and hydrogen peroxide. Any remaining $\mathrm{Cr} / \mathrm{Ir}$ was then removed by a high-pressure water stream in a mask cleaner.

Silicon nitride was deposited to a thickness of $1.0 \mu \mathrm{m}$ as the passivation layer for the probe. A $30 \mathrm{~nm}$ layer of chromium was deposited and patterned to later serve as the nitride etch mask. Fig. 3(a) depicts the cross-section at this stage.

Probe Shaping. A $15 \mu \mathrm{m}$ layer of AZ-P4620 photoresist (Shipley) was spun on to the wafers and patterned as a mask for the front-side plasma etches. The nitride over the trenches was etched using RIE with $15 \mathrm{sccm} \mathrm{CHF}_{3}$ and $4 \mathrm{sccm} \mathrm{O} \mathrm{O}_{2}$ at $80 \mathrm{~mW} / \mathrm{cm}^{2}$ (sufficient for a $-400 \mathrm{~V}$ bias) and $50 \mathrm{mTorr}$. The underlying silicon was plasma etched to a depth of $30-40 \mu \mathrm{m}$ in $100 \mathrm{sccm} \mathrm{SF_{6 }}$ and $70 \mathrm{sccm} \mathrm{C}_{2} \mathrm{ClF}_{5}$ at $260 \mathrm{~mW} / \mathrm{cm}^{2}$ and $100 \mathrm{mTorr}$.

After the photoresist was stripped, a $1 \mu \mathrm{m}$ layer of silicon nitride was deposited to protect the front of the wafer during the back side $\mathrm{KOH}$ etch. The wafer was diced in order to enhance the etching uniformity, then the dice were placed in a $15 \% \mathrm{KOH}$ solution at $80^{\circ} \mathrm{C}$. This solution thinned the silicon substrate from the back side at a rate of $1 \mu \mathrm{m} / \mathrm{min}$. A visual end condition was used, as the nitride in the trenches became transparent when the underlying silicon had been removed. After a few minutes overetch, the final probe thickness was $\approx 25 \mu \mathrm{m}$. See Fig. 3(b).

Finishing Steps. An RIE etch was used to strip the $\mathrm{KOH}$ barrier nitride. As the etch continued, portions of the passivation nitride layer were etched with the chromium layer

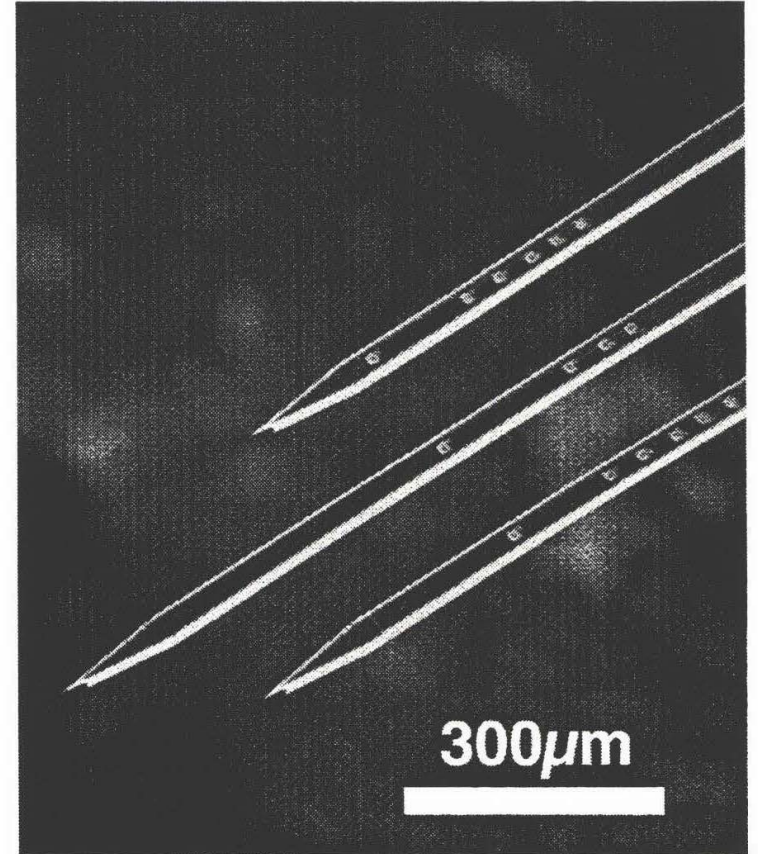

Figure 4. SEM of the tip region of a three-shaft probe, with 16 electrode sites: six on the left and right shafts, and four on the center shaft. The shafts are staggered in length.

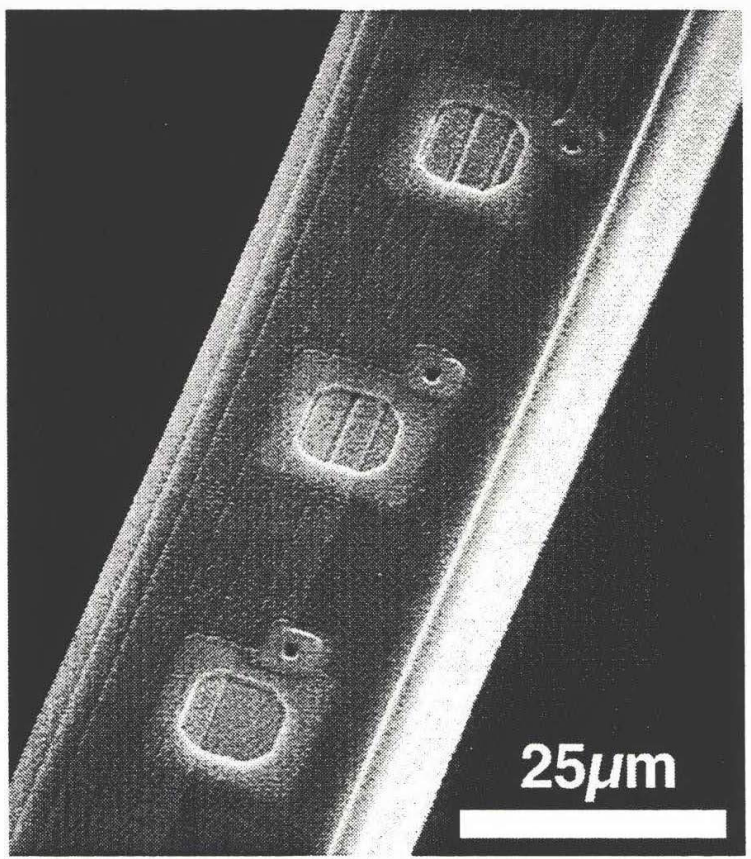

Figure 5. SEM of the top three electrode sites on a sixsite shaft. Note the interconnect traces running beneath the electrodes, and the small dimples where the metal-to-metal contacts are made.

serving as a mask, exposing the electrode sites and the bond pads. Finally the chromium itself was removed by immersion in a chromium etching solution. See Fig. 3(c).

The completed probes were mounted on PC boards, with the shafts projecting off the boards. After wire-bond connections were made between the probe and the boards, the 
probe base and wire bonds were encapsulated in an epoxy (87GT, Epoxy Technology, Inc).

\section{EXPERIMENTAL RESULTS}

Bench-Top Testing. SEMs of the neural probes are shown in Figs 4-6. Probe tip sharpness was evaluated in a SEM, which shows a tip radius of curvature of less than $0.25 \mu \mathrm{m}$. Preliminary impedance measurements indicate an electrode capacitance of $40 \mathrm{pF}$ with a shunt capacitance of $20 \mathrm{pF}$.

In gain measurements across 72 amplifier channels, midband gain was found to be $43.5 \pm 0.5 \mathrm{~dB}$. Total harmonic distortion was found to be less than $1 \%$ with a $5 \mathrm{mVpp}$ input and less than $0.25 \%$ with a $1 \mathrm{mVpp}$ input. The input-referred noise is $8 \mu \mathrm{V}$ rms for a bandwidth between 10 Hertz and $10 \mathrm{kHz}$. Operating at $\pm 2.5 \mathrm{~V}$ supplies, the entire chip dissipates $10 \mathrm{~mW}$.

Neural Recordings. The subjects for the physiological tests of the probe system were 90-180 day old female albino Sprague-Dawley rats, weighing about $250 \mathrm{~g}$. During an experiment, an animal was anesthetized with ketamine, xylazine, and acepromazine, together with a small initial dose of pentobarbital. Surgery was performed to allow access to a portion of the right somatosensory neocortex, which responds to touch stimuli. The exposed brain was continuously bathed in a warm saline solution. The animal was euthanized with an overdose of pentobarbital at the end of the experiment.

To make a recording, a probe assembly was mounted on a motorized micromanipulator, positioned over the exposed brain, and slowly inserted. The deepest electrode on one of the probe shafts was monitored during insertion using both an oscilloscope and an audio system. Recordings were made at locations where several of the electrodes were close enough to neurons to pick up clear neural activity. Signal quality on each channel was comparable to that obtained with a conventional extracellular microelectrode. See Fig. 7 for an example of the data recorded.

The neural recordings demonstrate that the probes and electronics work, and that they are capable of recording highquality data. These tests have also uncovered some areas where more work is needed. Despite the sharp tips, the probes still cause some dimpling, especially in experiments conducted in the rat cerebellum, where the pia is relatively tough. In one experiment, the pia was removed and a probe was inserted into the cerebellum with no dimpling, verifying that the pia is the major source of resistance to electrode entry.

Furthermore, although the probes have recorded action potentials in all preparations tried, some of which did not yield high-quality recordings. Besides neocortex, olfactory bulb slices (mitral cells) and deep portions of cerebellum (Golgi cells and deep Purkinje cells) have produced recordings with good signal-to-noise ratios. In the superficial cerebellum, the neonatal rat brainstem nuclei, and the locust antennal lobe, the signal quality obtained with probes has been worse than that typically obtained with conventional single electrodes.

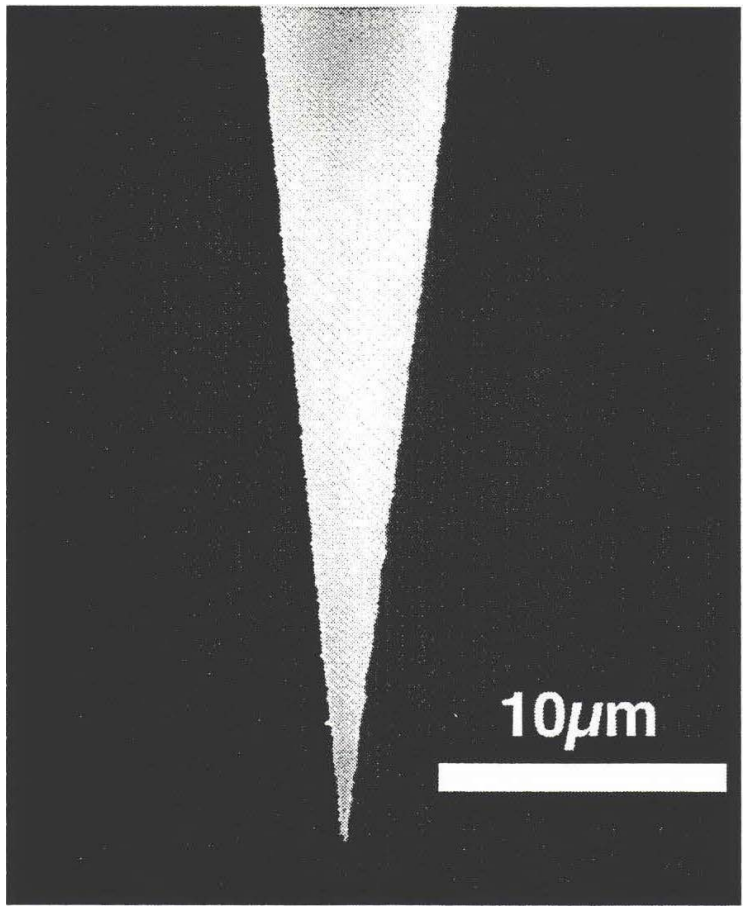

Figure 6. SEM of a probe tip (top view), showing the sharpness yielded by the dry etch process.

Another problem is that the probes are light sensitive. Photogenerated carriers in the probe substrate appear to couple capacitively into the gold traces via depletion regions formed at the surface of the silicon.

\section{CONCLUSION}

The positive results obtained so far indicate that the basic probe design is sound. It is believed that the probes are damaging nearby neurons during insertion, possibly because of a misalignment of the probe shafts to the insertion axis and snagging of the tissue by an abrupt edge in the nitride near the tips. The shaft misalignment is visually apparent and is probably due to stresses in the encapsulating adhesive lifting the probe shafts off the carrier PC board. New probe mounting methods are being explored to solve the adhesive stress problem, and the mask set will be redesigned to eliminate the nitride edge. The light sensitivity can be eliminated by placing a metal layer under the interconnect traces to provide electrical shielding from the substrate.

\section{ACKNOWLEDGMENTS}

This work has been funded by the National Science Foundation under grant \# PC147129-1. Additional support was provided by Medtronic, Inc. Karim Elaagouby, Mike Wehr, and Nick Mellen have contributed to this research by providing additional experiment preparations. 


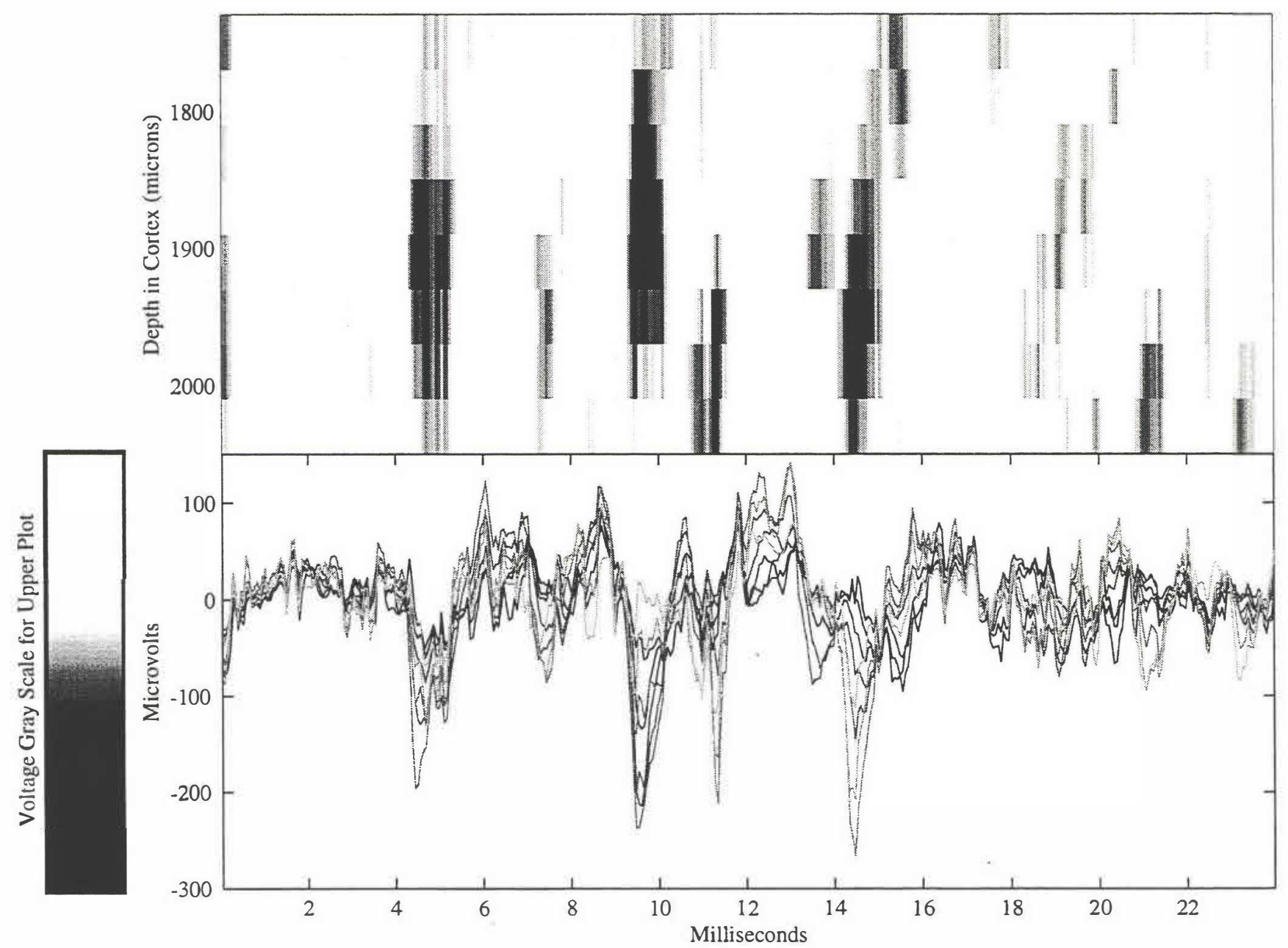

Figure 7. (Bottom) Eight channels of data obtained from one of two single shafts of a silicon probe in rat primary somatosensory cerebral cortex. The electrode sites were spaced vertically on the probe needle at $40 \mu \mathrm{m}$ intervals, covering $280 \mu \mathrm{m}$ of cortical depth. The data presented shows a spontaneous burst of activity recorded in an anesthetized rat. (Top) The same data plotted to show spiking with respect to time and the depth into the cortex. Darker regions indicate increased neural activity.

\section{REFERENCES}

1. S. A. Deadwyler and R. E. Hampson, "Ensemble Activity and Behavior: What's the Code?", Science, 270, 1316 (1995).

2. U. S. Bhalla and J. M. Bower, "Multi-Day Recordings from Olfactory Bulb Neurons in Awake Freely Moving Rats: Spatial and Temporal Patterns in Odorant Responses", Joumal of Computational Neuroscience (in press).

3. M. A. Wilson and B. L. McNaughton, "Reactivation of Hippocampal Ensemble Memories During Sleep”, Science, 265, 676 (1994).

4. M. A. L. Nicolelis, L. A. Baccala, R. C. S. Lin, and J. K. Chapin, "Sensorimotor Encoding by Synchronous Neural Ensemble Activity at Multiple Levels of the Somatosensory System", Science, 268, 1353 (1995).

5. K. D. Wise, J. B. Angell, and A. Starr, "An Integrated-Circuit Approach to Extracellular Microelectrodes", IEEE Transactions on Bio-Medical Engineering, BME-17, 238 (1970).
6. K. Najafi, K. D. Wise, and T. Mochizuki, "A High-Yield ICCompatible Multichannel Recording Array", IEEE Transactions on Electron Devices, ED-32, 1206 (1985).

7. L. D. Clark, "System for Chronic Neural Signal Transduction Processing and Control", Ph.D. dissertation, Massachusetts Institute of Technology (1990).

8. N. A. Blum, B. G. Carkhuff, H. K. Charles, Jr., R. L. Edwards, and R. A. Meyer, "Multisite Microprobes for Neural Recordings", IEEE Transactions on Biomedical Engineering, 38, 68 (1991).

9. M. Kuperstein and H. Eichenbaum, "Unit Activity, Evoked Potentials and Slow Waves in the Rat Hippocampus and Olfactory Bulb Recorded with a 24-Channel Microelectrode", Neuroscience, 15, 703 (1985).

10. E. Peeters, B. Puers, W. Sansen, J. Gybels, and P. DeSutter, "A Two-Wire, Digital Output Multichannel Microprobe for Recording Single-Unit Neural Activity", Sensors and Actuators B, 4, 217 (1991). 
11. R. S. Pickard, P. Wall, and M. Ubeid, "Recording Neural Activity in the Honeybee Brain with Micromachined Silicon Sensors", Sensors and Actuators B, 1, 460 (1990).

12. O. J. Prohaska, F. Pacha, P. Pfundner, and H. Petsche, "A 16-Fold Semi-Microelectrode for Intracortical Recording of Field Potentials", Electroencephalography and Clinical Neurophysiology, 47, 629 (1979).

13. K. Takahashi and M. Tadayuki, "Integration of MultiMicroelectrode and Interface Circuits by Silicon Planar and Three-Dimensional Fabrication Technology", Sensors and Actuators, 5, 89 (1984).

14. R. S. Pickard, "A Review of Printed Circuit Microelectrodes and Their Production", Journal of Neuroscience Methods, 1, 301 (1979).

15. D. J. Edell, V. V. Toi, V. M. McNeil, and L. D. Clark, "Factors Influencing the Biocompatibility of Insertable Silicon Microshafts in Cerebral Cortex", IEEE Transactions on Biomedical Engineering, 39, 635 (1992).
16. G. T.A. Kovacs, "Microelectrode Models for Neural Interfaces," pp 121-165 in D.A. Stenger, T.M. McKenna (Eds) (1994), "Enabling Technologies for Cultured Neural Networks," Academic Press, San Diego.

17. C. M. Gray, P. E. Maldonado, M. Wilson, and B. McNaughton, "Tetrodes Markedly Improve the Reliability and Yield of Multiple Single-Unit Isolation from Multiunit Recordings in Cat Striate Cortex", Journal of Neuroscience Methods, 63, 43 (1995).

18. G. T. A. Kovacs, C. W. Storment, M. Halks-Miller, C. R. Belczynski, Jr., C. DellaSantina, E. R. Lewis, and N. I. Maluf, "Silicon-Substrate Microelectrode Arrays for Parallel Recording of Neural Activity in Peripheral and Cranial Nerves", IEEE Transactions on Biomedical Engineering, 41, 567 (1994).

19. J. Ji and K. D. Wise, "An Implantable CMOS Circuit Interface for Multiplexed Microelectrode Recording Arrays", IEEE Journal of Solid State Circuits, 27, 433 (1992). 